\title{
A transient processing deficit following selection of an auditory target
}

\author{
TODD A. MONDOR \\ Mount Allison University, Sackville, New Brunswick, Canada
}

\begin{abstract}
Two experiments were conducted to examine the possibility that a transient processing deficit might result following selection of an auditory target. In both experiments, listeners were presented with a sequence of tones and required to make separate detection judgments, one regarding a target and the second regarding a subsequent probe. The target was presented following a variable number of tones, and the probe was presented in one of the seven or eight possible temporal positions following the target. The experiments differed only in that in Experiment 1 the first post-target item could be either a distractor tone or a probe, whereas in Experiment 2 the first post-target item was replaced by a silent period. In both experiments, a deficit in processing of the probe was apparent when it was presented in one of the first few positions following the target.
\end{abstract}

The question of whether the perception of auditory information might depend on the voluntary deployment of attention has historically been the subject of a great deal of empirical examination (e.g., Cherry, 1953; Creelman, 1959; Green, 1958; Moray, 1959, 1974; Treisman, 1960, 1969). These pioneering investigations established that voluntary attention to a location or to a frequency can facilitate the accuracy of perception of both verbal material and pure tones embedded in noise. Interest in auditory selective attention has been renewed recently, with several investigators examining how an advance cue might influence the perception of a subsequent target under conditions in which voluntary attentional deployment is unlikely. Notwithstanding occasional null results (e.g., Buchtel \& Butter, 1988; Posner, 1978), this research has generally both replicated and extended the earlier work in establishing that the speed and accuracy with which a response is made to a target might be either facilitated (e.g., Mondor \& Bregman, 1994; Mondor \& Zatorre, 1995; Mondor, Zatorre, \& Terrio, 1998; Spence \& Driver, 1994) or inhibited (Mondor, Breau, \& Milliken, 1998; Reuter-Lorenz, Jha, \& Rosenquist, 1996) by advance location and frequency cues.

Given that this previous research has established that the deployment of auditory attention might affect perception of a single auditory target, one obvious question is whether selection of the target might affect subsequent processing. Moray (e.g., 1974; Moray, Fitter, Ostry,

This paper is dedicated to the memory of M. Philip Bryden. This research was supported by grants from the Natural Sciences and Engineering Research Council of Canada and the Heart and Stroke Foundation of New Brunswick. I am indebted to Hal Pashler, Tram Neill, and Jane Raymond for their rigorous evaluation of an earlier version of this paper. The author's mailing address is Department of Psychology, Mount Allison University, Sackville, NB, Canada EOA 3CO (e-mail: tmondor@mta.ca).
Favreau, \& Nagy, 1976; Ostry, Moray, \& Marks, 1976) conducted a series of experiments to examine the effects of responding to an auditory target on subsequent processing. In these experiments, two channels of information (channels were usually defined by spatial location or frequency range) could be presented simultaneously and listeners were required to monitor both of these channels for a prespecified target (targets could be defined by either physical [e.g., frequency or intensity] or semantic [e.g., digits/numbers] features). Moray found that following a detection response to a target presented in one channel there was a temporary deficit in processing information in the other channel. A similar result was reported by Shaffer and Hardwick (1969), who found that this deficit persisted for approximately $1 \mathrm{sec}$ (see also Mowbray, 1964; Shaffer, 1968). These experiments yielded reliable evidence, then, that processes involved in selecting and responding to a target temporarily limited the ability of a listener to deal with new information. Because listeners made responses immediately following each target and were required to monitor two different channels of information, however, this processing deficit might be a function of several processes, including actual selection of the target, decision processes (including decisions related both to response selection and to switching attention between alternative channels), and interference produced by response execution. In discussing the results of his experiments, Moray (1974) argued that "when the observer recognizes a target and produces an overt 'yes' response his doing so interferes with processing of the contralateral signal," and because "this happens when a contralateral false alarm is made it is not due to the presence of a target, but to the making of a response" (both quotes from p. 131). Clearly, then, although these studies established a processing deficit subsequent to a target, the extent to which this reflects a limitation resulting from selection of the target is quite uncertain. 
The possibility that selection of a target might compromise subsequent processing has been investigated within the visual modality by several researchers (e.g., Broadbent \& Broadbent, 1987; Raymond, Shapiro, \& Arnell, 1992). In these studies, a rapid serial visual presentation (RSVP) technique is employed in which observers are presented with a sequence of stimuli (often letters) within which two different targets may be presented (the first of these is referred to as the target; the second is referred to as the probe). Following termination of a sequence, observers are typically required to make two different judgments: one regarding the target and one regarding the probe. It has been shown repeatedly that the accuracy with which a response is made to the probe depends critically on its temporal position relative to the target, with a marked processing deficit apparent when the probe occurs immediately following the target (e.g., Broadbent \& Broadbent, 1987; Raymond et al., 1992, 1995; Reeves \& Sperling, 1986; Shapiro \& Raymond, 1994; Shapiro, Raymond, \& Arnell, 1994; Weichselgartner \& Sperling, 1987). As Shapiro and Raymond (1994) wrote,

It is as if the perceptual and/or attentional mechanisms blink after target presentation. Eye blinks produce a dramatic, brief reduction in pattern vision and are only initiated after [italics in original text] important stimuli are viewed. The results of multiple-target RSVP studies just reviewed, as well as those we have conducted in our laboratory, suggest that sensory and/or attentional mechanisms may undergo a covert analogue to this overt ocular process. For this reason and for ease of reference, we have labelled the post-target processing deficit the attentional blink. (p. 159)

Thus, Shapiro and Raymond (1994) have argued that a visual attentional blink results directly because of the processes involved in selecting an object. Although a variety of theoretical explanations of this effect have been offered (some of these alternative explanations will be discussed in the preface to Experiment 2), the basic result is quite reliable. In several respects, the RSVP paradigm that has been used in studies of the visual attentional blink has two distinct advantages over that used by Moray (e.g., 1974) and others. First, the presentation of a single channel on each trial eliminates the possibility that performance might be affected by strategies related to switching attention between alternative channels--an affect documented by Moray (1974), who showed that the response criterion adopted for each channel was related to the relative probability of targets in the two channels. Second, because responses are made only at the end of a sequence, the possibility that interference resulting from response execution might account for the performance deficit may be safely rejected.

In the present study, an auditory version of the RSVP technique developed by Shapiro and Raymond (1994) was used to determine whether selecting an auditory target affected processing of subsequent auditory information. If such a result were in fact obtained, this would suggest that a transient processing deficit following selection might not be specific to vision, as Shapiro et al. (1994) have suggested, but rather that this is a general characteristic of human information processing.

\section{EXPERIMENT 1}

Experiment 1 was designed to address the possibility that a transient processing deficit follows selection of an auditory target. To this end, we attempted, insofar as is possible, to replicate the important design features used in studies of the visual attentional blink (e.g., Raymond et al., 1992). Participants were presented with a rapid sequence of sounds. Within each such sequence, a target and a probe could be presented, each with a probability of .50 . This design meant that sequences that included both a target and a probe, only a target, only a probe, or neither a target nor a probe were all equally likely. When presented, the target occurred following a variable number of regular nontarget (distractor) tones. The probe could occur only in one of the eight possible temporal positions subsequent to the target. Whereas the target and the distractors were all pure tones, the target differed from the distractors quantitatively on a single feature since it was of a much higher frequency. The probe, a complex tone that comprised five different frequencies, differed qualitatively from the target and the distractors (pure vs. complex). Listeners were required to make separate target and probe-detection judgments following termination of each sequence.

The target task was designed to be of moderate difficulty in order to increase the probability of identifying any probe processing deficit that might exist following target selection (Chun \& Potter, 1995). If selection of the target produces a temporary deficit in the processing of subsequent sounds, then performance on "probe-only" trials should be relatively stable across temporal positions, while a temporary deficit in probe-detection accuracy should be apparent on "target-plus-probe" trials.

\section{Method}

Thirty-two undergraduate students attending Mount Allison University participated as subjects in exchange for course credit. All had normal hearing according to self-report. Sounds were synthesized using the MITSYN signal-processing software package (Henke, 1992) at a sampling frequency of $32000 \mathrm{~Hz}$. All tones were $30 \mathrm{msec}$ in duration and had 5-msec linear onset/offset amplitude ramps to eliminate the occurrence of onset/offset clicks. Twenty-six pure tones were used as distractors. The frequencies of these were $\log$ related, ranging from 452 to $3462 \mathrm{~Hz}(452,493,537,586,639,697,760,829,904,944$, $986,1029,1122,1224,1335,1456,1587,1731,1888,2059,2245$, $2448,2670,2911,3175$, and $3462 \mathrm{~Hz}$ ). All tones could be easily differentiated from one another. A pure tone of $4000 \mathrm{~Hz}$ was synthesized for use as the target. Finally, a complex sound composed of five logrelated frequencies $(600,775,1002,1295$, and $1674 \mathrm{~Hz})$ was composed for use as a probe. Thus, the target differed quantitatively (frequency) and the probe, qualitatively (complex vs. pure) from the distractors.

The experiment was conducted using a $486 / 50$ IBM-compatible computer running the Maple programming environment (Bregman, Achim, \& Ahad, 1992). Sounds were presented binaurally through Sennheisser HD 250 Linear II headphones. On each trial, the subjects 
were presented with a sequence of between 16 and 24 tones. Interstimulus interval between consecutive pattern tones was set at $60 \mathrm{msec}$. Distractors were chosen randomly from the pool described above, such that there was no predictable frequency relation between notes. On $50 \%$ of the trials, a target was presented following between 7 and 15 pattern notes (randomly selected). Eight tones always followed the target. A probe occurred on $50 \%$ of trials, and when present could occur in any of these eight post-target positions. Thus, the target and the probe were each presented on $50 \%$ of trials. Listeners were required to make separate target- and probe-detection judgments following termination of each sequence. Judgments were made in response to questions that appeared on the computer screen (i.e., "Was the target present [press 1] or absent [press 0]?" followed by "Was the probe present [press 1] or absent [press 0]?"). The first question appeared $1 \mathrm{sec}$ following the last tone of the sequence; the second question appeared immediately following an acceptable response to the first question. Following these judgments, listeners initiated the subsequent trial by pressing any key on the computer keyboard. The firs note of the next trial began $750 \mathrm{msec}$ following this initiating keypress. Prior to the experiment, the target and the probe were presented to the subjects in isolation in order to familiarize them with the discriminations they would be required to perform. In addition, the subjects completed at least 24 practice trials. With regard to the practice trials, each subject was required to meet a criterion of $60 \%$ correct on the probe task before beginning the experimental trials. None of the listeners required more than two sets of 24 practice trials to meet this criterion. All subjects completed 192 experimental trials.

\section{Results and Discussion}

Target detection. The subjects found the targetdetection task relatively difficult as, averaged across all conditions, the probability of a correct response to the target task was $0.75(S E=0.024)$. The probability of a correct response on target-present trials was 0.861 , and the probability of a false-positive response on probe-present trials was $0.36(S E=0.03) . d^{\prime}$ on probe-present trials averaged $1.68(S E=0.162)$.

Probe detection. A two-way repeated measures analysis of variance (ANOVA) (target presence $\times$ probe position) was conducted using the conditional probability of accurate probe detection given a correct target-detection response as the dependent variable. This analysis revealed significant main effects of both target presence $[F(1,31)=$ $21.20, p<.001]$ and probe position $[F(7,217)=5.45$, $p<.001]$. In general, listeners more accurately detected the probe when the target was absent and when it occurred toward the end of the sequence. The interaction between target presence and probe position, described in the top panel of Figure 1, also reached significance $[F(7,217)=$ $3.83, p<.001]$. This interaction resulted because of a significant effect of probe position when the target was present $[F(7,217)=6.34, p<.001]$ but not when it was absent $[F(7,217)=1.53, p=.16]$. In accordance with prior research (e.g., Shapiro \& Raymond, 1994), the existence of a processing deficit was evaluated by directly comparing, for each probe position, detection performance on target-present and target-absent trials. These planned comparisons revealed significant probe-processing deficits on target-present trials at the $+1,+2,+3,+5$, and +6 positions ( $p<.05$ for all comparisons). Thus, these results establish the existence of a transient processing deficit or "auditory blink" when listeners must select and process a target.

Even though false-positive rates did not differ as a function of whether or not the target was present $(p>.10$; $.082, S E=0.020$, and $.094, S E=0.020$, for target-present and target-absent trials, respectively), $d^{\prime}$ was examined to determine whether the auditory blink might have resulted from differences in response bias for target-present and target-absent trials. ${ }^{1}$ This analysis was entirely consistent with the analysis of percent correct. Specifically, main effects of target presence $[F(1,31)=20.13$, $p<.001]$ and probe position $[F(7,217)=4.17, p<.001]$ reached significance, as did the target presence $\times$ probe position interaction $[F(7,217)=2.33, p<.05]$. The interaction arose because of a significant effect of probe position on target-present trials $[F(7,217)=4.53, p<.001]$ but not on target-absent trials $[F(7,217)=1.14, p=.34]$.

\section{EXPERIMENT 2}

In Experiment 2, the effect of replacing the +1 item by silence on the magnitude of the auditory blink was examined as a means of evaluating the applicability to audition of several of the theories that have been proposed as explanations of the visual attentional blink. Shapiro and Raymond (1994; Raymond et al., 1995) have offered a model that is based largely on the attentional engagement theory of Duncan and Humphreys $(1989,1992)$. According to this model, an attentional template operates to determine whether any particular item in the stimulus sequence will be allowed entry into a limited-capacity visual short-term memory store (VSTM). This template is defined according to the relevant features of the task. Thus, in a typical "visual blink" experiment, the template would hold a description of both the target and the probe. Each item in the sequence would be evaluated with respect to the template and only those that closely matched the template description would be passed on to VSTM. Each time an item is passed into VSTM, it is likely that the item immediately following it will "slip in" as well. The number of items held in memory therefore will depend in part on whether or not the observer was required to select both a target and a probe. According to this model, the visual attentional blink results because of interference between items held in memory at the time of response selection. When only a probe is presented "the number of items gaining access to VSTM is reduced and no interference effects should be observed. When both target and probe tasks must be performed, additional items gain access to VSTM and interference effects should ensue" (Shapiro \& Raymond, 1994, p. 175). Furthermore, if the +1 item is replaced by a silent period, then the observed processing deficit should be markedly attenuated because of a reduction in the number of items, and the amount of interference, in STM.

Other explanations of the transient processing deficit apparent following selection of a visual target have been 

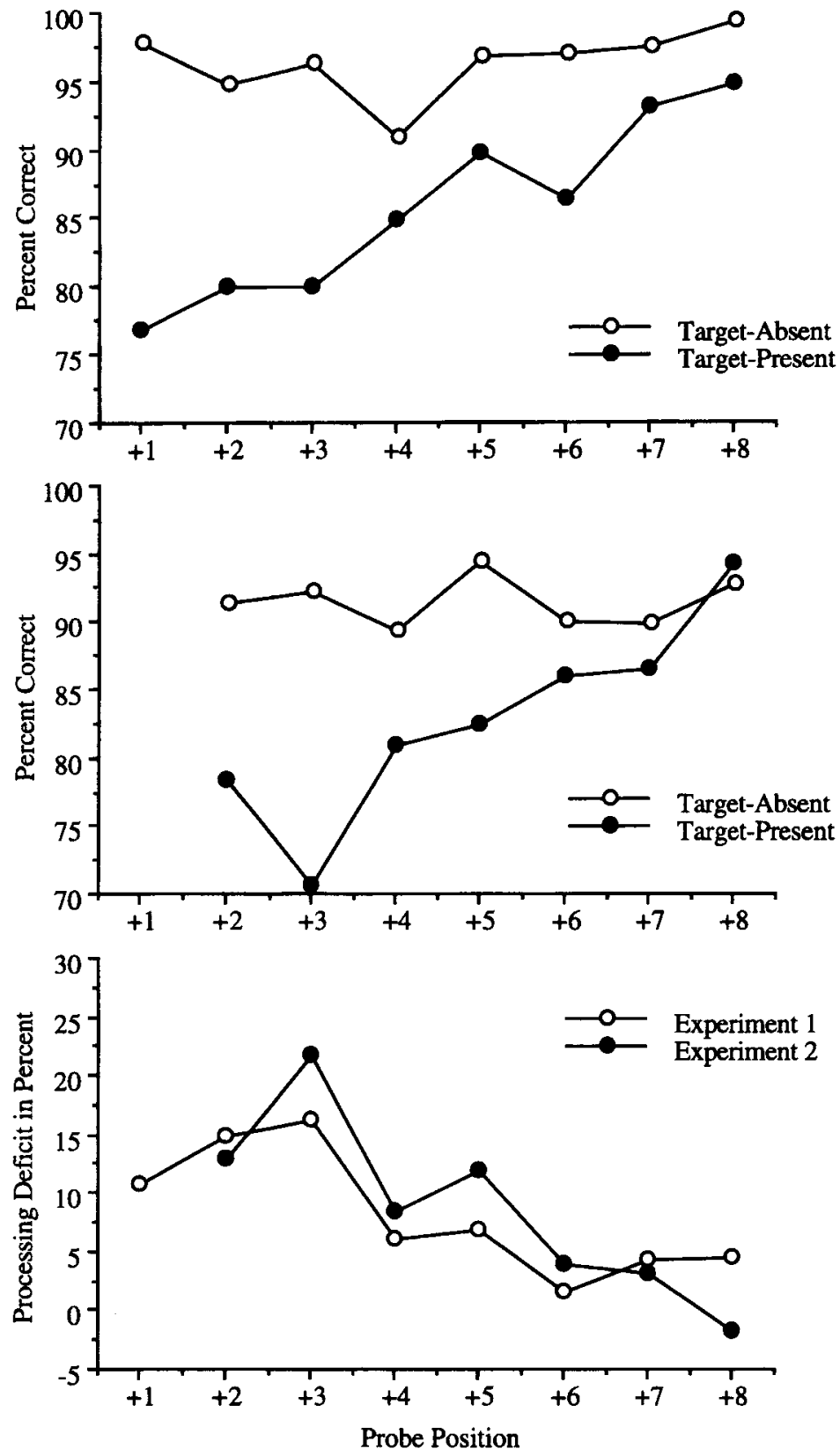

Figure 1. Probe-detection accuracy for target-absent and target-present trials as a function of probe position is described for Experiment 1 (top panel), and for Experiment 2 (middle panel). In the bottom panel, the magnitude of the processing deficit on target-present trials as a function of probe position is shown for both experiments.

offered. Chun and Potter (1995) argued that the visual attentional blink arises primarily because of a limitedcapacity target "identification and consolidation" processing stage that follows an initial stage in which the target is detected. A "blink" results because of a delay in processing probes that occurs while Stage 2 is occupied with dealing with the target. According to this model, local similarities (e.g., between the target and the +1 item) affect the difficulty of target identification and thereby the dura- tion of Stage 2. Therefore, increasing the difficulty of target discrimination acts to increase the magnitude of the attentional blink because of the increased duration of Stage 2. According to this model, then, replacement of the +1 item by silence should lead to a reduction in the magnitude of the processing deficit.

Finally, Raymond et al. (1992) proposed that the blink results because of an inhibitory process that acts to prevent items subsequent to the target from interfering with 
target processing. This inhibitory suppression mechanism, or attentional gate, is activated by the presence of stimuli subsequent to the target. Therefore, when the +1 position contains stimulus information, the suppression mechanism is engaged and a temporary processing deficit is produced. However, when no stimulus information is presented in the +1 position, no deficit should occur.

Although the theoretical proposals of Shapiro and Raymond (1994), Raymond et al. (1992), and Chun and Potter (1995) are quite different from one another, all predict that replacement of the +1 item by silence should either eliminate altogether or reduce the magnitude of the processing deficit (albeit for different reasons). Critically, with regard to visual processing, this common prediction has been empirically verified by both Chun and Potter (1995, Experiment 3) and Raymond et al. (1992, Experiment 3).

Investigation of the psychological refractory period (PRP) suggests an alternative explanation for the auditory blink. The PRP, which is operationally defined as a delay in responding to the second of two successive stimulus events (e.g., Telford, 1931), has been studied extensively (e.g., Bertelson, 1967; Netick \& Klapp, 1994; Osman \& Moore, 1993; Pashler, 1993, 1994a). While several theoretical accounts have been offered, it appears that a processing bottleneck at response selection is of primary importance in producing the PRP (Pashler, 1994b; Pashler \& Johnston, 1989; see also McCann \& Johnston, 1992, who argued on the strength of results from a dualtask paradigm that the bottleneck in human information processing is at the level of response selection). The delay in responding to the second of two targets, which has been documented in studies of the PRP, and the processing deficit apparent in Experiment 1 appear to be at least somewhat similar in that in both cases two responses must be selected. This similarity raises the possibility that the "auditory blink" may result because of a processing limitation at the level of response selection. In considering this as a possible explanation of the visual attentional blink, Shapiro and Raymond (1994) argued that the "PRP account" was unlikely to be correct because, as noted above, the visual blink is eliminated when the +1 item is replaced by a blank interval (Raymond et al., 1992). As Shapiro and Raymond reasoned, the possibility that the visual blink occurs because of a limitation at response selection appears to be inconsistent with any significant effect of the +1 item because "it does not seem possible that a blank interval could affect such a stage, given that the two stimuli that clearly require response selection (target and probe) are still present in the same temporal relation to one another" (p. 162).

Experiment 2 was designed to determine whether elimination of the +1 item would affect the transient processing deficit observed in Experiment 1 . To this end, the +1 item was replaced by a silent period. If, under these conditions, no performance deficit for probe detection on target-present trials is apparent, the possibility that the auditory blink might result because of either inter- ference (Chun \& Potter, 1995; Shapiro \& Raymond, 1994) or inhibition (Raymond et al., 1992) would receive some support. In contrast, if a processing deficit is again observed in spite of this modification, the applicability of these models as explanations to the auditory blink will have been cast into doubt and the possibility that the deficit arises because of a processing bottleneck at response selection will receive some support.

\section{Method}

Thirty-three undergraduate students attending Mount Allison University participated as subjects in exchange for course credit. None of the subjects reported any hearing impairment. The computer and sound system and the sounds used were identical to those used in Experiment 1 . In design and procedure, Experiment 2 was identical to Experiment 1 except that the eighth position from the end of the sequence (the +1 item position for target-present trials) was replaced by a 30-msec silence on every trial. Thus, probes could occur only in the +2 to +8 positions. The subjects completed 28 practice and 168 experimental trials.

\section{Results and Discussion}

In spite of detecting the probe on the practice trials at a level better than $60 \%$ correct, probe-detection accuracy on the experimental trials fell to $47 \%$ for one subject. The data obtained from this subject were dropped from all analyses.

Target detection. Subjects found the target-detection task somewhat less difficult than in Experiment 1, with the probability of a correct response to the target task increasing to 0.813 ( $S E=0.021)$. Performance on targetpresent and probe-present trials increased to $0.92(S E=$ 0.013 ), and the probability of false positive declined to 0.28 on probe-present trials $(S E=0.026) . d^{\prime}$ on probepresent trials increased to $2.22(S E=0.150)$

Probe detection. A two-way ANOVA (target presence $X$ probe position) using the conditional probability of an accurate probe-detection response given a correct response to the target task as the dependent variable revealed significant main effects of target presence $[F(1,31)=$ $13.17, p<.001]$ and probe position $[F(6,186)=7.92, p<$ $.001]$, as well as an interaction between the two $[F(6,186)=$ $3.79, p<.01]$. The interaction resulted because, although performance improved with probe position for both target-present $[F(6,186)=7.71, p<.001]$ and targetabsent trials $[F(6,186)=2.49, p<.05]$, the effect was more robust when a target was presented. Planned comparisons revealed significantly reduced probe-detection accuracy on target-present trials relative to target-absent trials at the $+2,+3,+4$, and +5 positions $(p<.05$ for all comparisons). As may be seen in the middle panel of Figure 1, the results of Experiment 2 are quite consistent with those of Experiment 1 in indicating the existence of a transient processing deficit following auditory target selection in spite of the fact that an extended silent period followed the target.

False-positive rates did not differ as a function of whether or not the target was present $(p>.10 ; 0.158$, $S E=0.029$, and $0.135, S E=0.029$, for target-present and target-absent trials, respectively). As was apparent in Ex- 
periment 1, an ANOVA of $d^{\prime}$ was consistent with the results obtained from the percent-correct analysis with significant main effects of target presence $[F(1,31)=10.03$, $p<.01]$ and probe position $[F(7,217)=7.25, p<.001]$ and a significant interaction between target presence and probe position $[F(7,217)=3.21, p=.05]$. A significantly larger increase in $d^{\prime}$ as a function of probe position was apparent on target-present trials $[F(7,217)=7.56, p<.001]$ than on target-absent trials $[F(7,217)=2.66, p<.05]$.

Evidence of a transient processing deficit following target presentation was again obtained even though the +1 item was replaced by silence. As outlined above, such a result is inconsistent with the possibility that the auditory blink occurs because of interference between items in memory, or because of a delay in, or inhibition of, processing the probe. In contrast, the obtained deficit is consistent with the possibility that the auditory blink occurs because of a processing bottleneck at response selection.

\section{CONCLUDING COMMENTS}

A transient processing deficit following target selection was apparent in both Experiment 1 and Experiment 2. Critically, this deficit was observed even when the +1 item was replaced by silence. As discussed above, this later effect is inconsistent with the interference (Chun \& Potter, 1995; Shapiro \& Raymond, 1994) and inhibition (Raymond et al., 1992) theoretical explanations of the visual attentional blink that have been offered recently. All of these models predict that eliminating stimulus information from the +1 position should attenuate the magnitude of the deficit. No evidence was obtained in support of this possibility, inasmuch as a statistical comparison of performance in the two experiments revealed no significant differences. ${ }^{2}$

The stability of the attentional blink across experiments, shown in the bottom panel of Figure 1, is consistent with the possibility that the auditory blink results because of a processing bottleneck at response selection. This single result is, of course, not definitive, and both this and other possible explanations should be evaluated further. For example, in both experiments, the listener was required to switch tasks from searching for the target to searching for the probe (when both were present). Although the results of several studies of visual information processing suggest that task preparation does not contribute significantly to either the visual attentional blink (Chun \& Potter, 1995) or the PRP (e.g., Pashler, 1994b), this does not necessarily mean that task preparation is irrelevant with respect to producing an auditory blink. If task switching does contribute to the auditory blink, then the magnitude of the processing deficit might be expected to be related to the similarity of the target and probe with the magnitude of the blink decreasing as target-probe similarity increases. Other factors, such as local similarities between the target and the immediately preceding distractor as well as global target-distractor similarities might also be important determinants of the auditory blink and are worth pursuing empirically.

The fact that a transient processing deficit following target selection is apparent in both audition and vision might be taken as evidence of a basic limitation in human information processing. The similarity in the duration of the processing deficit for visual (approximately 200-500 msec according to Chun \& Potter, 1995) and auditory information (approximately $450-540 \mathrm{msec}$ in Experiments 1 and 2) lends some support for this possibility. However, there appear to be two striking differences between the auditory and visual blinks. First, findings by Raymond et al. (1995) and by Chun and Potter (1995) that the similarity of the target to the +1 item affects the magnitude of the visual attention blink suggest that the visual processing deficit does not result because of a processing limitation at response selection. Second, the linear decrease in the magnitude of the auditory blink as a function of temporal position of the target, which was apparent in Ex- periment 1 (and to a lesser extent in Experiment 2), is rather different from the U-shaped function that has typically been reported for the visual blink (e.g., Chun \& Potter, 1995; Raymond et al, 1992). Both Chun and Potter and Raymond et al. (1992) interpreted the advantage for probes in the +1 position over those in the +2 and +3 positions as an indication that the +1 item was not delayed or inhibited because it was selected and processed along with the target. Differences in the magnitude of the visual and auditory blinks as a function of temporal position of the probe and the apparent differential effect of the nature of the +1 item on the blinks may, therefore, be indicative of important differences in the cognitive mechanisms responsible for these effects.

Clearly, issues such as the relation between the auditory and visual blinks remain to be resolved. However, the two experiments reported above are important for two reasons. First, they establish the existence of a transient processing deficit following selection of an auditory target. Second, because this deficit was unaffected by the elimination of the +1 item, it appears more likely the result of a processing bottleneck at response selection rather than of either interference or inhibition.

\section{REFERENCES}

BERTELsON, P. (1967). The refractory period of choice reactions with regular and irregular interstimulus intervals. Acta Psychologica, 27, 45-56.

Bregman, A. S., Achim, A., \& Ahad, P. A. (1992). The Maple software system [Computer software]. Montreal: McGill University.

Broadbent, D. E., \& Broadbent, M. H. P. (1987). From detection to identification: Response to multiple targets in rapid serial visual presentation. Perception \& Psychophysics, 42, 105-113.

BuChTEL, H. A., \& BUTTER, C. M. (1988). Spatial attention shifts: Implications for the role of polysensory mechanisms using visual and tactile stimuli. Neuropsychologia, 26, 499-509.

CHERRY, E. C. (1953). Some experiments on the recognition of speech, with one and with two ears. Journal of the Acoustical Society of America, 25, 975-979.

Chun, M. M., \& PotTer, M. C. (1995). A two-stage model for multiple target detection in rapid serial visual presentation. Journal of Experimental Psychology: Human Perception \& Performance, 21, 109-127.

Creelman, C. D. (1959). Detection of signals of uncertain frequency. Journal of the Acoustical Society of America, 32, 805-810.

DunCAN, J., \& HumphreYs, G. W. (1989). Visual search and stimulus similarity. Psychological Review, 96, 433-458.

DunCan, J., \& Humphreys, G. W. (1992). Beyond the search surface: Visual search and attentional engagement. Journal of Experimental Psychology: Human Perception \& Performance, 18, 578-588.

GreEN, D. M. (1958). Detection of multiple component signals in noise. Journal of the Acoustical Society of America, 30, 904-911.

HENKE, W. L. (1992). An interactive dialogue language for time signal processing [Computer software]. Cambridge, MA: MIT Research Laboratory of Electronics.

MCCANN, R. S., \& JoHNSTON, J. C. (1992). Locus of the single-channel bottleneck in dual-task interference. Journal of Experimental Psychology: Human Perception \& Performance, 18, 471-484.

Mondor, T. A., Breau, L. M., \& Milliken, B. (1998). Inhibitory processes in auditory selective attention: Evidence of locationbased and frequency-based inhibition of return. Perception \& Psychophysics, 60, 296-302.

MondoR, T. A., \& BREgMAN, A. S. (1994). Allocating attention to frequency regions. Perception \& Psychophysics, 56, 268-276.

MONDOR, T. A., \& ZATORRE, R. J. (1995). Shifting and focusing auditory spatial attention. Journal of Experimental Psychology: Human Perception \& Performance, 21, 387-409.

Mondor, T. A., Zatorre, R. J., \& Terrio, N. A. (1998). Constraints on the selection of auditory information. Journal of Experimental Psychology: Human Perception \& Performance, 24, 66-79.

Moray, N. (1959). Attention in dichotic listening: Affective cues and the influence of instructions. Quarterly Journal of Experimental Psychology, 12, 214-220.

MORAY, N. (1974). A data base for theories of selective listening. In 
P. M. A. Rabbitt \& S. Dornic (Eds.), Attention and performance $V$ (pp. 119-135). New York: Academic Press.

Moray, N., FitTer, M., Ostry, D., Favreau, D., \& NaGy, V. (1976). Attention to pure tones. Quarterly Journal of Experimental Psychology, 28, 271-283.

Mowbray, G. H. (1964). Perception and retention of verbal information presented during auditory shadowing. Journal of the Acoustical Society of America, 36, 1459-1464.

Netick, A., \& KLAPP, S. T. (1994). Hesitations in manual tracking: A single-channel limit in response programming. Journal of Experimental Psychology: Human Perception \& Performance, 20, 766-782.

OSMAN, A., \& MOORE, C. M. (1993). The locus of dual-task interference: Psychological refractory effects on movement-related brain potentials. Journal of Experimental Psychology: Human Perception \& Performance, 19, 1292-1312.

Ostry, D., Moray, N., \& Marks, G. (1976). Attention, practice, and semantic targets. Journal of Experimental Psychology: Human Perception \& Performance, 2, 326-336.

PASHLER, H. (1993). Dual-task interference and elementary mental mechanisms. In D. E. Meyer \& S. Kornblum (Eds.), Attention and performance XIV (pp. 245-264). Cambridge, MA: MIT Press.

PASHLER, H. (1994a). Dual-task interference in simple tasks: Data and theory. Psychological Bulletin, 116, 220-244.

PASHLER, H. (1994b). Overlapping mental operations in serial performance with preview. Quarterly Journal of Experimental Psychology, 47A, 161-191.

Pashler, H., \& Johnston, J. C. (1989). Chronometric evidence for central postponement in temporally overlapping tasks. Quarterly Journal of Experimental Psychology, 41A, 19-45.

PosNer, M. I. (1978). Chronometric explorations of mind. Hillsdale, $\mathrm{NJ}$ : Erlbaum.

Raymond, J. E., Shapiro, K. L., \& ARnell, K. M. (1992). Temporary suppression of visual processing in an RSVP task: An attentional blink? Journal of Experimental Psychology: Human Perception \& Performance, 18, 849-860.

Raymond, J. E., Shapiro, K. L., \& Arnell, K. M. (1995). Similarity determines the attentional blink. Journal of Experimental Psychology: Human Perception \& Performance, 21, 653-662.

ReEVES, A., \& SPERLING, G. (1986). Attentional gating in short-term visual memory. Psychological Review, 93, 180-206.

Reuter-Lorenz, P. A., JHA, A. P., \& RosenQuist, J. N. (1996). What is inhibited in inhibition of return? Journal of Experimental Psychology: Human Perception \& Performance, 22, 367-378.

SHAFFER, L. H. (1968). Refractoriness in information processing. Quarterly Journal of Experimental Psychology, 20, 38-50.

SHAFFER, L. H., \& HARDWICK, J. (1969). Monitoring simultaneous auditory messages. Perception \& Psychophysics, 6, 401-404.
Shapiro, K. L., \& Raymond, J. E. (1994). Temporal allocation of visual attention: Inhibition or interference. In D. Dagenbach \& T. H. Carr (Eds.), Inhibitory mechanisms in attention, memory and language (pp. 151-187). San Diego: Academic Press.

Shapiro, K. L., Raymond, J. E., \& ARNell, K. M. (1994). Attention to visual pattern information produces the attentional blink. Journal of Experimental Psychology: Human Perception \& Performance, 20, 357-371.

SPENCE, C. J., \& DRIVER, J. (1994). Covert spatial orienting in audition: Exogenous and endogenous mechanisms. Journal of Experimental Psychology: Human Perception \& Performance, 20, 555-574.

TELford, C. W. (1931). The refractory phase of voluntary and associative responses. Journal of Experimental Psychology, 14, 1-36.

Treisman, A. M. (1960). Contextual cues in selective listening. Quarterly Journal of Experimental Psychology, 12, 242-248.

Treisman, A. M. (1969). Strategies and models of selective attention. Psychological Review, 76, 282-299.

WeichselgartNer, E., \& Sperling, G. (1987). Dynamics of automatic and controlled visual attention. Science, 238, 778-780.

\section{NOTES}

1. Note that a false-positive response cannot be associated specifically with one probe position. Therefore, $d^{\prime}$ calculations for each of the probe positions on target-present (target-absent) trials was performed using the overall probability of a false-positive response on target-present (target-absent) trials

2. Because the probability of a false-positive detection response to the probe was somewhat higher in Experiment 2 than in Experiment 1, cross-experiment statistical comparisons were conducted using both $d^{\prime}$ and percent correct. Separate three-way mixed ANOVAs (experiment $X$ target presence $X$ probe position) of probe-detection performance (probe-present trials only) for the +2 to +8 positions revealed for both measures (for each effect, the statistical result from the $d^{\prime}$ analysis is reported first followed by the corresponding result from the percentcorrect analysis) significant main effects of target presence $[F(1,62)=$ $24.02, p<.001 ; F=26.75, p<.001]$ and probe position $[F(6,372)=$ $10.25, p<.001 ; F=8.30, p<.001]$ and an interaction between target presence and probe position $[F(6,372)=3.67, p<.01 ; F=5.11, p<$ $.001]$. The main effect of experiment $[F(1,62)=4.78, p<.05 ; F=3.30$, $p=.07]$ indicated that performance was better in Experiment 1 . None of the other effects approached significance ( $p>.22$ in all cases).

(Manuscript received February 5, 1997; revision accepted for publication November $7,1997$. 